\section{Interferon $\beta$-1a subcutaneously 3 times/week clinical outcome in relapsing multiple sclerosis in Finland}

\author{
Elina Järvinen,1 Annukka Murtonen,2 \\ Melina Tervomaa, 3 \\ Marja-Liisa Sumelahti ${ }^{1}$ \\ 1Merck Finland, Espoo; Department of \\ Medicine, University of Helsinki; \\ ${ }^{2}$ Faculty of Medicine and Life Sciences, \\ University of Tampere; 3 StatFinn, \\ Espoo, Finland
}

\begin{abstract}
Prognostic factors and long-term treatment response of interferon $\beta$-1a s.c tiw has not been studied in a real-life clinical cohort in Finland. The aim of the paper was to evaluate long-term treatment response, prognostic clinical factors and adherence among interferon $\beta$-1a s.c tiw treated ment adherence among patients treated with interferon $\beta$-1a s.c. tiw in Finland. Interferon $\beta$-1a s.c. tiw is $100 \%$ reimbursed by Finnish Social Security Insurance (SII) from 1996 in active relapsing MS and used with doses of 22 and $44 \mu \mathrm{g}$ tiw. Patients who were initially treated with interferon $\beta$ 1a s.c. tiw during the follow-up period from 1996 to 2010 are included in the study.
\end{abstract} patients in Finland. A retrospective review of medical records was performed. Confirmed relapsing multiple sclerosis patients treated with interferon $\beta$-1a s.c tiw $22 \mu \mathrm{g}$ or $44 \mu \mathrm{g}$ as their first treatment, from 1996 to 2010 in Western Finland, were included. Longitudinal generalized linear regression models were applied to assess risk of disability progression, using Expanded Disability Status Scale (EDSS), during the treatment period. Odd's ratios with $95 \%$ confidence intervals $(95 \% \mathrm{CI})$ were calculated for risk factors: gender, age at diagnosis, treatment delay, dose, baseline EDSS and EDSS change in one year. Kaplan-Meier was applied to study median time to discontinuation. Mean duration of treatment in 293 cases was 2.9 years (min 0.04, max 13.5). EDSS increase vs. no increase in one-year carried a significant risk for long-term disability progression (1.20, 1.08-1.33). Older age, defined by a 10 -year increase in age at diagnosis (1.43, $1.07-1.91)$ and one-year delay to treatment start showed an increased risk for disability progression $(1.05,0.99-1.11)$, but gender $(0.66,0.38-1.15)$ or initial dose $(1.00,0.45$ 2.25) showed no risk. Treatment was stopped in $37 \%$ due to disease activation at median of 1.7 years, and in $25 \%$ due to side effects at 9.3 months. Our results show that young age, a short delay to treatment start and slower disability progression were identified as factors for better outcome among cases with interferon $\beta$-1a s.c tiw as their first disease modifying treatment.

\section{Introduction}

The increasing number of approved treatments for relapsing multiple sclerosis (MS) shows varying levels of efficacy and safety. ${ }^{1}$ To date, there are more than a dozen approved treatments for MS, including the injectable therapies, which are generally considered as a safe treatment option. ${ }^{2}$ The optimal treatment success is still challenging and there is not yet consensus on the best treatment strategy or treatment target in MS. ${ }^{3}$ Prognostic factors for long-term clinical outcomes are needed to help in treatment management. 4

The aim of the present study is to assess the long-term clinical outcome and treat-

\section{Materials and Methods}

The National Institute for Health and Welfare and the local ethical standards committee approved the retrospective examination of identified patient records. Cases in this retrospective long-term real-life study are confirmed relapsing MS patients diagnosed by using Poser and McDonald criteria during 1981-2010 at the neurological hospital clinics, which belong to Tampere University Central Hospital districts in Western Finland.5,6 Patients who initiated interferon $\beta$-1a s.c tiw $22 \mu \mathrm{g}$ or $44 \mu \mathrm{g}$ during $1996-2010$ as their first disease modifying treatment (DMT) were included in the study. The follow-up period of the study was from 1996 to 2010.

Data was collected for gender, age at diagnosis, time from disease onset to treatment initiation, time from diagnosis to treatment initiation, treatment duration from treatment start to cessation. Disability progression was estimated by using longitudinal Expanded Disability Status Scale (EDSS) measures. ${ }^{7}$ In the longitudinal analysis the EDSS value was always compared to the previous reported EDSS value. Therefore, the change is dependent on the previous value and not on the baseline value. Initial EDSS value at treatment start (baseline EDSS), yearly EDSS change during the treatment, and causes for discontinuation were collected from hospital patient charts by authors AM and MLS.
Correspondence: Marja-Liisa Sumelahti, University of Tampere, Faculty of Medicine and Life Sciences, 33014 Tampere, Finland. E-mail: marja-liisa.sumelahti@staff.uta.fi

Key words: Multiple sclerosis, Interferon $\beta$-1a s.c tiw, Disability progression, EDSS.

Acknowledgements: This study was sponsored by Merck Finland, Helsinki, Finland (An affiliate of Merck KGaA, Darmstadt, Germany). The authors would like to thank Pasi Korhonen (Stat Finn) and Melina Tervomaa (Stat Finn) for help in statistical analysis and Pedro Testi (Merck Research Clinical Operations) for coordination of the study.

Contributions: MLS: Collection of material, design of the study, analysis and interpretation of the data, drafting the manuscript. EJ: Design of the study, interpretation of the data, drafting the manuscript. AM: Collection of material, drafting the manuscript.

Conflict of interest: EJ is an employee of Merck Finland, an affiliate of Merck KGaA, Darmstadt, Germany.

Funding: Funding for this work was provided by Merck Finland, an affiliate of Merck KGaA, Darmstadt, Germany.

Received for publication: 13 May 2019. Accepted for publication: 24 October 2019.

This work is licensed under a Creative Commons Attribution NonCommercial 4.0 License (CC BY-NC 4.0).

(C) Copyright: the Author(s), 2019

Licensee PAGEPress, Italy

Neurology International 2019; 11:8177

doi:10.4081/ni.2019.8177

Treatment reimbursement requirements for DMTs in MS in Finnish SII are active disease and description of patients' disability status. This and patients' status information were available from hospital patient records. Disability was assessed by using EDSS in majority of the patient records and in case it was described authors (AM, MLS) applied EDSS scale retrospectively based on recorded status information.

Generalized linear regression models were used to assess Odd's ratios (OR) with 95\% confidence intervals (95\% CI) for covariates: gender, age at diagnosis, time from diagnosis to treatment start, dose of interferon $\beta$-1a s.c tiw, baseline EDSS and EDSS change in one year. For statistical analyses baseline EDSS values were categorized into three groups: EDSS 0-1, EDSS $1.5-2.5$ and EDSS 3.0 or greater. 
Kaplan-Meier (K-M) curve with Log Rank test was used to study the difference in the times on treatment in groups categorized based on the cause of discontinuation. Causes of discontinuation were classified into three groups as follows: 1 . Side effects included flu like reactions, any medication related laboratory value change or injection related skin problems, 2. MS activation, in case patient experienced a new relapse or disability progression was observed, 3. Other causes: Neutralizing antibodies, at six months (180 days or more) after medication start, lack of adherence or discontinuation due to economical causes, pregnancy (gravidity or a pregnancy plan).

\section{Results}

A total of 293 patients, female/male ratio $207 / 86$, started interferon $\beta$-1a. s.c tiw as first treatment during the follow-up. At treatment start (baseline) mean age was 36.4 years. The time from disease onset to treatment initiation was 6.3 years and the time from diagnosis to treatment initiation was 2.8 years. Mean baseline EDSS was 1.7. (Table 1). Altogether 290 EDSS values from a total of 179 patients were recorded during the follow-up. Distribution of cases in baseline EDSS categories shows majority in group 0-1.0: $55.6 \%(\mathrm{n}=163)$, in $1.5-2.5$ $17 \%(\mathrm{n}=50)$ and in $>3.027 .3 \%(\mathrm{n}=80)$. No gender difference existed in baseline EDSS categories (Chi sq 0.88). Compliance to regular treatment visits was good and yearly information for disability was available in all included cases. Five cases of death were recorded during the follow-up period and in three cases treatment was used up to patients' death; deaths were not related to interferon $\beta$-1a s.c tiw.

Mean duration of the first treatment (doses $22 \mu \mathrm{g}$ or $44 \mu \mathrm{g}$ ) was 2.9 years (SD 2.9 , min 0.04 , max 13.5 years), no difference was observed between baseline EDSS groups ( $\mathrm{F}$ value $0.98, \mathrm{P}<0.001$ ). Disease activation in $37.3 \%(\mathrm{n}=77)$ and side effects $25.2 \%(n=52)$ were the main causes of discontinuation in the majority of the 209 cases, excluding deaths $(n=3)$. Side effects were injection related $(n=12)$, other side effects $(n=37)$ and flu like symptoms $(n=3)$. Other causes were neutralizing antibodies in $15.4 \%(\mathrm{n}=31)$, pregnancy in $9.7 \% \%$ $(n=20)$, no disease activity $3.4 \% \quad(n=7)$, other causes $9.2 \%(n=19)$. Total of 84 cases were on treatment at the end of follow-up in 2010. Mean time on treatment among these cases was 3.98 years (min 0.04, max 13.5 years). Figure 1 shows K-M curve for the time in years from interferon $\beta$-1a. s.c tiw start to discontinuation by discontinuation cause. Median time to discontinuation in case of any side effects $(n=52)$ was 9.3 months (280 days), activation of disease

( $\mathrm{n}=77) 1.7$ year (20.5 months; 616 days) and other causes $(n=77) 2.3$ years $(28.1$ months; 843 days). EDSS values in yearly

Table 1. Characteristics of the study population.

\begin{tabular}{|c|c|c|}
\hline & No. & $\%$ \\
\hline Total & 293 & 100 \\
\hline Men & 86 & 29.4 \\
\hline Women & 207 & 70.6 \\
\hline Interferon $\beta$-la s.c tiw $22 \mu \mathrm{g}$ & 252 & 86.0 \\
\hline \multirow[t]{2}{*}{ Interferon $\beta$-la s.c tiw $44 \mu g$} & 41 & 14.0 \\
\hline & Mean, Median & SD \\
\hline Age at diagnosis, years & $33.6,32.0$ & 9.5 \\
\hline Age at treatment initiation, years & $36.4,36.0$ & 10.1 \\
\hline Time to treatment from onset, years & $6.3,4.0$ & 6.8 \\
\hline Time from diagnosis to treatment, years & $2.8,1.0$ & 4.6 \\
\hline EDSS at treatment initiation & $1.7,1.0$ & 1.6 \\
\hline Duration of treatment period, years & $2.9,1.9$ & 2.9 \\
\hline
\end{tabular}

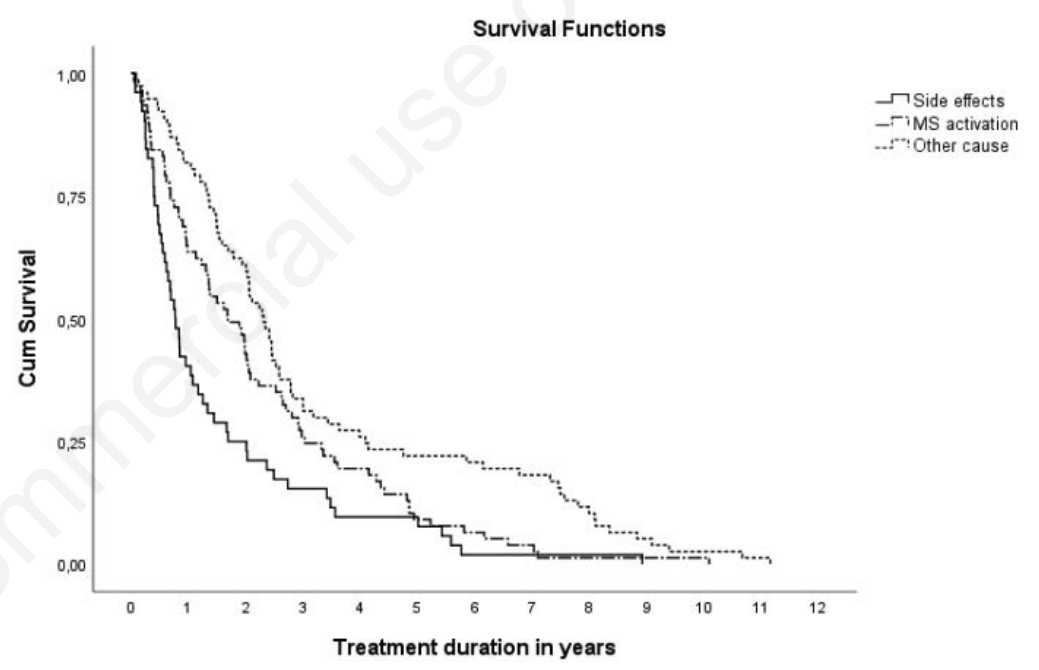

Figure 1. Time from interferon $\beta$-1a. s.c tiw start to discontinuation by cause. K-M curve. Time to discontinuation (years) of treatment $(n=206)$ in groups: Side effects $(n=52)$, MS activation $(n=77)$ and other causes $(n=77)$.

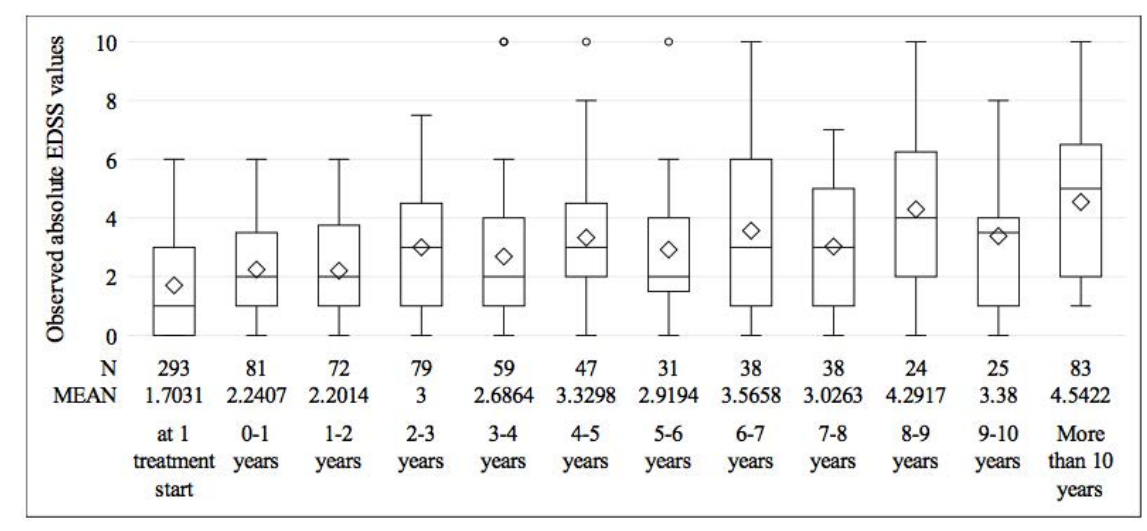

Figure 2. Number of MS cases on $\beta$-1a. s.c tiw, observed absolute, mean and median EDSS values and SD in yearly intervals from baseline at start during follow-up period from 1996 to 2010. 
intervals by treatment duration are given in Figure 2. The mean EDSS points at mild disability (3.0 or less) during the first 3 years of treatment.

Results in the longitudinal analysis are shown in Figure 3. A statistically increased risk for disease progression related to onepoint one-year increase in EDSS (1.20, 1.08-1.33). Ten-year increase in age at diagnosis increased the risk for progression significantly $(1.43,1.07-1.91)$. High baseline EDSS value $(>3)$ as compared to a low baseline EDSS (0-1.0) value showed a statistically significant lower risk for disease progression assessed by EDSS increase $(0.25,0.10-0.63)$. No statistically significant risk was shown for gender $(0.66,0.38$ $1.15)$ or initial dose of interferon $\beta-1$ a s.c tiw $(1.00,0.45-2.25)$. Yearly delay from diagnosis to treatment start was close to statistical significance $(1.05,0.99-1.11)$ (Figure 3 and Supplementary Table 1).

\section{Discussion and Conclusions}

We studied clinical outcomes in relapsing MS cases with interferon $\beta$-1a s.c tiw as the first treatment during the 14-year follow-up from 1996 to 2010 in neurological hospital clinics in Western Finland. A total of 293 confirmed relapsing-remitting MS cases fulfilling the Finnish SII reimbursement criteria were included. We observed a good compliance to treatment, based on the accurate information in patient records at regular yearly visits. Disability status by EDSS was assessed at yearly visits by the treating neurologist. At interferon $\beta$-1a s.c. tiw start the mean baseline EDSS was 1.7, mean age 36.4 years and time to treatment initiation 2.8 years from diagnosis. The observed long delay reflects the variable diagnostic year from 1981 to 2010 in this cohort, where in some cases diagnosis was assessed years before the availability of interferon $\beta$-1a s.c. tiw in 1996.

EDSS scale is considered a sensitive tool to assess changes in disease progression. ${ }^{8}$ However, some studies have shown that the rates of change vary depending on the initial value, and several reports point at higher annual rates of change (disability progression) at low EDSS baseline but little change from EDSS score of 6 onwards.9,10 These observations are important when disability progression is determined by EDSS during the treatment. In our data a low EDSS 0-1.0 carried a greater progression risk as compared to EDSS $>3.0$, evaluated by increase in EDSS score in the longitudinal study. The prognostic benefit, however, related strongly to a younger age, pointing at the significance of early treatment initiation, which was sup- ported by the negative impact for increasing the time to treatment initiation. Considering the limitations regarding the use of EDSS scale, the risk for disease progression, however, related to one-point increase in yearly EDSS, which corroborates results in other interferon- $\beta$ studies. ${ }^{11,12}$ Here no risk related to gender, supporting the results that there is no indication of different effects of interferon- $\beta$ in men or women. ${ }^{13}$ The result that the initial dose of interferon showed no effect on the risk, is likely due to the differences in the number of patients in the groups, and overall low number of patients especially in the 44 $\mu \mathrm{g}$ group.

The mean duration of the first treatment with interferon $\beta$-1a s.c. tiw was almost three years. The treatment periods thus remained relatively short for deciphering the long-term treatment responses. Disability progression reached a mean EDSS 5.0 from baseline for cases who had stayed on treatment up to 10 years or more, reflecting a moderate disability at this point. A total of 84 cases were on interferon $\beta$-1a s.c. tiw treatment at the end of follow-up in 2010 . During the 14-year follow-up the treatment was stopped in $70 \%$. Disease activation at mean of 1.7 years $(37.3 \%)$ and side effects at mean of 9.3 months $(25.2 \%)$ were the main causes. Percentage for stopping interferon- $\beta$ treatment in another study was only $18 \%$ during 6-year- follow up (mean 3.5 \pm 2.1 years), where the main reason for treatment discontinuation was lack of efficacy (54\%) and $21 \%$ of patients stopped therapy because of pregnancy and only $17 \%$ because of AE.14 Differences as compared to our results may be explained by a longer followup in our cohort. Furthermore, disease activity and progression in interferon $\beta$-1a s.c tiw treated cases today may be detected early after baseline by using MRI. 15 No evidence of disease activity (NEDA), also referred to as freedom from disease activity, is a new goal that is emerging in multiple sclerosis treatment. Earlier treatment discontinuation as observed here may be expected in future cohorts.

Treatment discontinuation in case of neutralizing antibodies leads to treatment change in Finland. The percentage of patients with neutralizing antibodies in our cohort was $15 \%$, while in the PRISMS study the percentages were $23.8 \%$ in $22 \mu \mathrm{g}$ and $12.5 \%$ in $44 \mu \mathrm{g} .16$ Efficacy of interferon- $\beta$-1a has been shown to decrease with neutralizing antibody formation, highlighting the importance of neutralizing antibodies to clinical response. ${ }^{17,18}$ The improved formulation of interferon $\beta$-1a s.c tiw, which has been available since 2007, was shown to reduce the number of patients developing neutralizing antibodies, and its introduction to clinical praxis is likely the cause for the lower percentage seen also here. ${ }^{19}$ Similar percentages have been reported in more recent studies. ${ }^{20}$

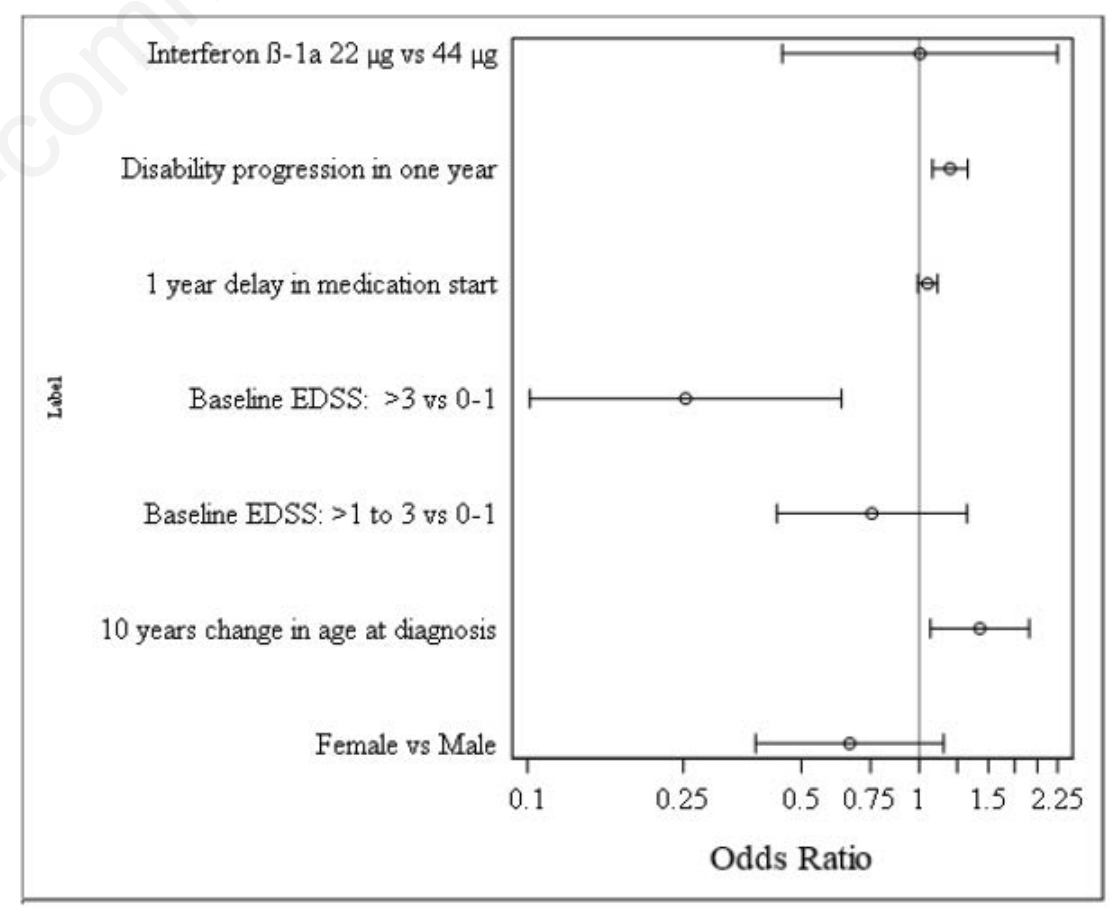

Figure 3. Longitudinal analysis showing OR's with $95 \%$ confidence intervals by clinical prognostic variables. Disability progression risk is increased if $\mathrm{OR}>1.0$ during the treatment period. 
Interferon $\beta$-1a s.c tiw was discontinued also in case of pregnancy or planned pregnancy. Due to large amount of data, which was collected from registries and post-marketing experience on pregnancies during interferon $\beta$-1a treatment, a label change for interferon $\beta$-1a treatments was recently done. Currently, if clinically needed, the use of interferon $\beta$-1a s.c tiw may be considered during pregnancy, and interferon $\beta-1 \mathrm{a}$ therapy can be used during breast-feeding. ${ }^{21,22}$ Therefore, interferon $\beta$-1a tiw may be useful among fertile young women wishing to become pregnant.

Real-life experience and reports on over 15 years of long-term use of interferon $\beta$-1a tiw has shown good safety profiles. ${ }^{2}$ Here the side effects weakened the treatment adherence and lead to discontinuation in about a quarter of cases, which is lower than the $35.7 \%$ reported in i.m interferon $\beta-1 \mathrm{a}$ community-based cohort $(n=364) .{ }^{23}$ In our study common side effects were injection site side effects, flu like reactions and increase in laboratory values, while no serious side effects were observed, which is in line with previous reports. ${ }^{16,23}$ Experience on patient education on these side effects prior to treatment, and proper management of adverse events improves adherence to treatment and these actions to diminish side-effects are needed to reach long-term treatment tolerance with interferon $\beta$-1a s.c. tiw. 24,25

The prognostic factors recognized in this study point at young age, a short time to treatment initiation, and slower disability progression during the follow up. There is a need for regular clinical follow-up to control for disease activity and a need for continuous patient education to improve treatment adherence.

\section{References}

1. Giovannoni G. Disease-modifying treatments for early and advanced multiple sclerosis: a new treatment paradigm. Curr Opin Neurol. 2018;31:2333.

2. Jakimovski D, Kolb C, Ramanathan M, et al. Interferon $\beta$ for Multiple Sclerosis. Cold Spring Harb Perspect Med. 2018;8:11.

3. Smith AL, Cohen JA, Hua LH. Therapeutic Targets for Multiple Sclerosis: Current Treatment Goals and Future Directions. Neurotherapeutics 2017;14:952-60.
4. Heesen C, Scalfari A, Galea I. Prognostic information for people with MS: Impossible or inevitable? Mult Scler 2018;16:1352458518807101.

5. Poser CM, Paty DW, Scheinberg L et al. New diagnostic criteria for multiple sclerosis: guidelines for research protocols. Ann Neurol 1983;13:227-1.

6. McDonald I, Compston A, Edan G et al. Recommended diagnostic criteria for multiple sclerosis: guidelines from the panel on the diagnosis of multiple sclerosis. Ann Neurol 2001;50:121-7.

7. Kurtzke JF. Rating neurologic impairment in multiple sclerosis: an expanded disability status scale (EDSS). Neurology 1983;33:1444-2

8. Hutchinson J, Hutchinson M. The Functional Limitations Profile may be a valid, reliable and sensitive measure of disability in multiple sclerosis. J Neurol 1995;242:650-7.

9. Ravnborg M, Blinkenberg M, Sellebjerg F et al. Responsiveness of the Multiple Sclerosis Impairment Scale in comparison with the Expanded Disability Status Scale. Mult Scler 2005;11:81-4.

10. Hohol MJ, Orav EJ, Weiner HL. Disease steps in multiple sclerosis: a longitudinal study comparing disease steps and EDSS to evaluate disease progression. Mult Scler 1999;5:349-4.

11. Kappos L, Kuhle J, Multanen J et al. Factors influencing long-term outcomes in relapsing-remitting multiple sclerosis: PRISMS-15. J Neurol Neurosurg Psychiatry 2015;86:1202-7.

12. Traboulsee AL, Cornelisse P, SandbergWollheim M, et al. Prognostic factors for long-term outcomes in relapsingremitting multiple sclerosis. Mult Scler J Exp Transl Clin 2016;2:205521 7316666406.

13. Magyari M, Koch-Henriksen N, Laursen B, Sørensen PS. Gender effects on treatment response to interferon-beta in multiple sclerosis. Acta Neurol Scand 2014;130:374-9.

14. Mesaroš S, Stojsavljević N, DujmovićBašuroski I, et al. Long-term adherence to interferon-beta treatment in a cohort of RRMS patients in Belgrade, Serbia. Clin Neurol Neurosurg 2012;114:11458 .

15. Traboulsee A, Li DKB, Cascione M, et al. Effect of interferon beta-1a subcutaneously three times weekly on clinical and radiological measures and no evidence of disease activity status in patients with relapsing-remitting multiple sclerosis at year 1. BMC Neurol 2018;18:143.

16. PRISMS (Prevention of Relapses and Disability by Interferon beta-1a Subcutaneously in Multiple Sclerosis) Study Group. Randomised double-blind placebo-controlled study of interferon beta-1a in relapsing/remitting multiple sclerosis. Lancet 1998;352:1498-4.

17. Paolicelli D, D'Onghia M, Pellegrini F, et al. The impact of neutralizing antibodies on the risk of disease worsening in interferon $\beta$-treated relapsing multiple sclerosis: a 5-year post-marketing study. Neurol 2013;260:1562-8.

18. PRISMS-4: Long-term efficacy of interferon-[beta]-1a in relapsing MS. pg. 1628-1636 DOI:10.1212/WNL.56.12.1628 The PRISMS (Prevention of Relapses and Disability by Interferon--1a Subcutaneously in Multiple Sclerosis) Study Group; the University of British Columbia MS/MRI Analysis Group. Neurology 2001;56:12.

19. De Stefano N, Curtin F, Stubinski B, et al. IMPROVE Study Investigators. Rapid benefits of a new formulation of subcutaneous interferon beta-1a in relapsing-remitting multiple sclerosis. Mult Scler 2010;16:888-92.

20. Comi G, De Stefano N, Freedman MS, et al. Subcutaneous interferon $\beta-1 \mathrm{a}$ in the treatment of clinically isolated syndromes: 3-year and 5-year results of the phase III dosing frequency-blind multicentre REFLEXION study. J Neurol Neurosurg Psychiatry 2017;88:285-4.

21. European Medicines Agency. Rebif [Summary of product characteristics]. 2019.

22. Cohan S, Chen C, Baraban E, et al. Results of sustained long-term use of interferon beta-1a in a communitybased cohort of patients with relapsing multiple sclerosis. J Drug Assess 2015; 4:1-6.

23. Bayas A, Ouallet JC, Kallmann B, et al. Adherence to, and effectiveness of, subcutaneous interferon $\beta$ - $1 \mathrm{a}$ administered by RebiSmart ${ }^{\circledR}$ in patients with relapsing multiple sclerosis: results of the 1year, observational SMART study. Expert Opin Drug Deliv 2015;12:123950.

24. Walther EU, Hohlfeld R. Multiple sclerosis: side effects of interferon beta therapy and their management. Neurology 1999;53:1622-7. 